\title{
Hemobilia, una causa infrecuente de hemorragia del tracto digestivo superior
}

\section{Hemobilia, a rare cause of upper gastrointestinal bleeding}

Mariana Ramírez,,$^{*}$ 미 Jasson Restrepo-Castrillón. ${ }^{2}$

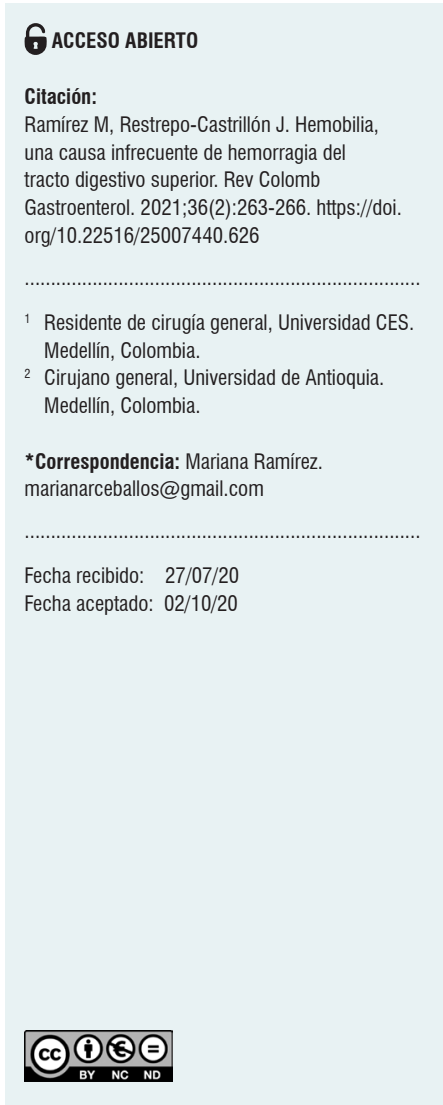

\begin{abstract}
Resumen
La hemobilia es una causa poco frecuente de hemorragia del tracto gastrointestinal superior. La principal etiología es de origen iatrogénico y la posibilidad de hemobilia debe considerarse en cualquier paciente con hemorragia gastrointestinal y un historial reciente de procedimientos hepatobiliares. Otras causas menos frecuentes incluyen el trauma de abdomen, la enfermedad oncológica de la vía biliar o las enfermedades inflamatorias del páncreas o la vía biliar. La presentación clínica varía según la gravedad del sangrado; generalmente se presenta con dolor abdominal, ictericia y melenas, aunque puede cursar al ingreso con rectorragia e hipotensión. Un alto porcentaje de estas presenta resolución espontánea, sin requerir procedimientos adicionales. La angiografía es el estándar de oro para el diagnóstico de la hemobilia, pero los avances en la angiotomografía permiten que esta sea una opción menos invasiva y con mayor disponibilidad. La angioembolización es el tratamiento principal para estos pacientes, pero existen otras alternativas como la colocación de stent vascular o de stent en el conducto biliar.
\end{abstract}

\section{Palabras clave}

Hemobilia, sangrado gastrointestinal, vía biliar.

\begin{abstract}
Hemobilia is a rare cause of upper gastrointestinal $(\mathrm{GI})$ tract bleeding. Its main etiology is iatrogenic, and the possibility of hemobilia should be considered in any patient with GI bleeding and a recent history of hepatobiliary surgery. Other less frequent causes include abdominal trauma, oncologic disease of the biliary tract, or inflammatory diseases of the pancreas or bile duct. Its clinical presentation varies depending on the severity of the bleeding. It usually presents with abdominal pain, jaundice, and tarry stools, although patients may also present with rectorrhagia and hypotension on admission. A high percentage of these symptoms have a spontaneous resolution, without requiring additional procedures. Angiography is the gold standard for the diagnosis of hemobilia, but advances in computed tomography angiography make it a less invasive and more widely available option. Endovascular embolization is the main treatment for these patients, but there are other alternatives such as vascular or bile duct stent placement.
\end{abstract}

\section{Keywords}

Hemobilia; Gastrointestinal bleeding; Biliary tract.

\section{CASO CLÍNICO}

Se trata de una paciente femenina de 68 años, con antecedente de diabetes mellitus e hipertensión arterial, quien ingresó por un cuadro clínico de dolor abdominal en el hipocondrio derecho asociado con ictericia, por lo que se le realizó una ecografía de abdomen que mostró colelitiasis y dilatación de la vía biliar. Se consideró que presentaba 
alto riesgo de coledocolitiasis y, entonces, se programó para colangiopancreatografía retrógrada endoscópica (CPRE), en la que se evidenció una imagen radiolúcida de gran tamaño en el colédoco, compatible con un síndrome de Mirizzi grado IV; no se logró la extracción endoscópica del cálculo, por lo que se decidió la inserción de un stent biliar y, además, se obtuvo drenaje purulento (Figura 1).

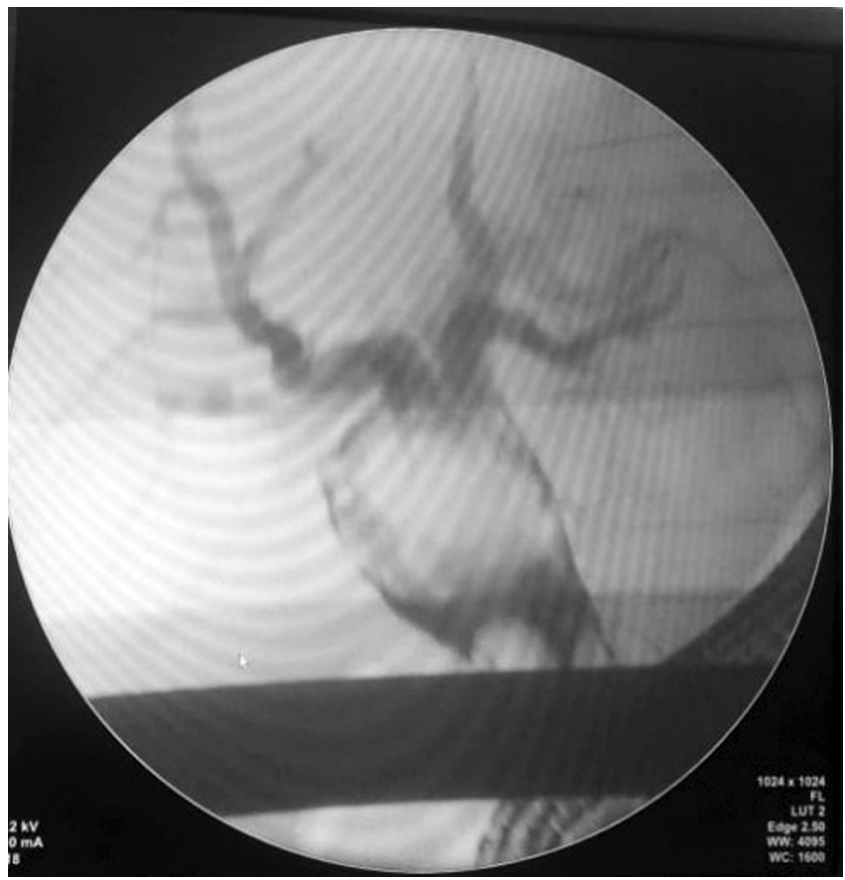

Figura 1. CPRE. Imagen radiolúcida en el colédoco, compatible con un síndrome de Mirizzi grado IV.

Posterior a esto, se programó para colecistectomía abierta con exploración de vías biliares. En el procedimiento se evidenció la vesícula con paredes engrosadas, con síndrome de Mirizzi grado IV; se realizó la extracción de un gran cálculo de la vía biliar mediante el uso de pinzas de Randall, se realizó un lavado exhaustivo de la vía biliar y se decidió dejar un tubo en T. Presentó una evolución tórpida durante el posoperatorio temprano, por lo que al tercer día se decidió realizar una tomografía axial computarizada (TAC) de abdomen en la que se evidenció una colección perihepática anterior, la cual es drenada de manera percutánea con éxito; y se obtuvo salida de material bilioso. A los 3 días del drenaje presentó abdomen agudo, por lo que requirió una reintervención, en la que se evidenció bilioperitoneo y tubo en $\mathrm{T}$ desalojado; se evacuó el líquido peritoneal y se realizó una coledocorrafia con parche de epiplón. Se trasladó a la unidad de cuidados intensivos (UCI) con soporte ventilatorio y vasopresor; allí se documentó la aparición de Klebsiella pneumoniae y Enterobacter cloacae en el líquido peritoneal, que fueron manejados con antibiótico dirigido. En los días siguientes presentó hematemesis y caída de la hemoglobina, por lo que fue llevada a endoscopia, en la que se evidenció un sangrado a través de la papila; por tanto, se realizó el manejo con escleroterapia, sin lograr control del sangrado; entonces se realizó una angiotomografía de abdomen en la que se encontró un pseudoaneurisma de la arteria hepática derecha con comunicación a la vía biliar (Figura 2).

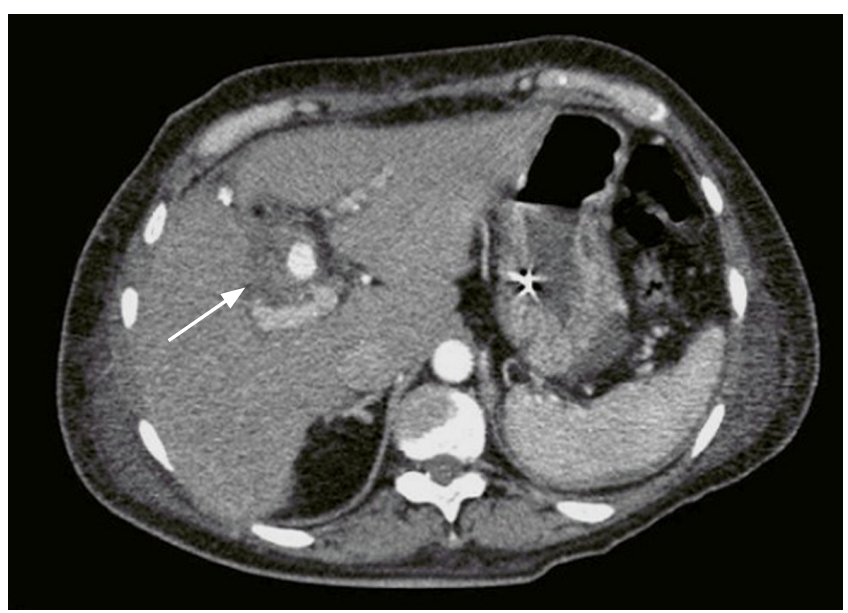

Figura 2. Angiotomografía de abdomen. Pseudoaneurisma de la arteria hepática derecha. Área hipodensa que rodea el vaso en contacto con la vía biliar (flecha).

Se solicitó una valoración por radiología intervencionista, en la que se realizó embolización con Onix de pequeñas ramas de la arteria hepática derecha. La paciente continuó con sangrado, asociado con hipotensión y requerimiento de soporte vasopresor, por lo que fue llevada a nueva arteriografía urgente del tronco celíaco, y supraselectiva de arteria hepática derecha con intento de embolización del pseudoaneurisma hepático, el cual no fue exitoso. Debido a este resultado, se realizó una embolización percutánea del pseudoaneurisma, con evidencia fluoroscópica posterior de la corrección del mismo, sin complicaciones. La paciente presentó una adecuada evolución sin sangrado ni anemización y sin requerir intervenciones adicionales.

\section{DISCUSIÓN}

La hemobilia es definida como un sangrado del tracto biliar y es una causa poco frecuente de hemorragia gastrointestinal alta, que corresponde a menos del $5 \%$ de todos los casos (1). El primer caso fue descrito en 1654 por Francis Glisson, un anatomista británico, que lo reportó posterior a un trauma cerrado en el cuadrante superior derecho del abdomen. El término hemobilia es utilizado desde 1948, cuando Philip Sandblom lo emplea en pacientes con dicho sangrado. A lo 
largo de los años se han reportado series de casos de hemobilia posterior a trauma, procedimientos endoscópicos o percutáneos; y su diagnóstico es más fácil cuando los pacientes presentan alguno de estos antecedentes, dado que por su poca incidencia la sospecha clínica es baja $(2,3)$.

La etiología es variable y ocurre cuando se presenta una fístula entre un vaso sanguíneo y la vía biliar. La obstrucción o lesión de los órganos del tracto pancreatobiliar pueden ser los causantes de esta. Normalmente, están involucrados vasos arteriales de alta presión, que lleva a tener sangrados abundantes, aunque están descritos casos en los cuales se presenta hipertensión portal y los directos causantes son vasos venosos (4). La proximidad anatómica de los conductos biliares y la vasculatura de la vía biliar los hace más propensos a lesiones iatrogénicas, por lo que aproximadamente en el $50 \%$ de los casos la hemobilia va a estar relacionada con procedimientos invasivos percutáneos, endoscópicos o quirúrgicos. La literatura reporta que el $38 \%$ de los pacientes con hemobilia son posteriores a drenajes percutáneos de la vía biliar o a biopsias hepáticas percutáneas, el $19 \%$ corresponde a procedimientos quirúrgicos tanto abiertos como laparoscópicos y el 10 $\%$ son procedimientos endoscópicos. Durante los procedimientos quirúrgicos, las lesiones biliovasculares pueden generarse por el paso de la sutura, diatermia, por los clips o por el instrumental que lesiona las paredes de los vasos y de la vía biliar. Se han descrito otras etiologías menos frecuentes como trauma, principalmente en trauma cerrado, neoplasias, patología biliar litiásica, patologías inflamatorias, infecciones por parásitos o malformaciones anatómicas $(5,6)$.

En cuanto a la fisiopatología, es importante resaltar que la mezcla entre la bilis y la sangre crea una dispersión entre las partículas que impide la adecuada formación de coágulos, por lo que estos son débiles y con poca adhesión. Además, se considera que la bilis contiene factores fibrinolíticos que limitan la producción de la fibrina. Se considera que, cuando un sangrado es abundante, este pasa al duodeno y se manifiesta con melenas o hematemesis; mientras que en los sangrados menores se pueden formar coágulos débiles que obstruyen la vía biliar, generan ictericia y estos, a su vez, predisponen a la posterior formación de cálculos (7).

Sus manifestaciones clínicas dependen de la cantidad y frecuencia del sangrado. La tríada de Quincke (cólico biliar, ictericia y sangrado) solo está presente en un tercio de los casos (8). El sangrado se manifiesta como melenas en el $90 \%$ y hematemesis en el $60 \%$, mientras que la ictericia y el dolor en el hemiabdomen superior están presentes en $60 \%$ y $70 \%$ de los casos, respectivamente. En algunos casos, la hemobilia se ha asociado con pancreatitis o colangitis. Se describe en pocos pacientes la presencia de anemia crónica, dadas las pérdidas sanguíneas secundarias a hemobilia (9). Cuando la hemobilia es secundaria a trauma, puede presentarse inmediatamente después del trauma o semanas después, y gene- ralmente el origen es $50 \%$ intrahepático y $50 \%$ extrahepático, con presencia de dolor abdominal en la mayoría de los casos, pero con aparición variable de ictericia (6).

El diagnóstico de hemobilia es complejo y se requiere una alta sospecha diagnóstica dada su baja incidencia. Siempre se debe sospechar en el paciente con hemorragia del tracto digestivo superior, con antecedente de instrumentación o manipulación de la vía biliar, o en aquellos que tienen diagnóstico previo de enfermedad biliopancreática. Los exámenes de laboratorio iniciales incluyen un cuadro hemático y un perfil hepático completo (transaminasas, bilirrubina y sus diferenciales), debido a que un importante porcentaje de los pacientes se presenta con anemia e hiperbilirrubinemia; además, es importante solicitar tiempos de coagulación $(10,11)$. El enfoque inicial del sangrado digestivo incluye un estudio endoscópico, con evidencia de sangrado o salida de coágulos a través de la ampolla de Vater hasta en el $90 \%$ de los pacientes. La CPRE es útil cuando la hemobilia está asociada con ictericia obstructiva, porque evidencia el sangrado por la papila y permite evacuar coágulos causantes de la obstrucción $(12,13)$.

Ante la sospecha diagnóstica de hemobilia, se realizaba con frecuencia la angiografía en busca de anormalidades vasculares como pseudoaneurismas o fístulas que explicaran el sangrado, lo que permitía la corrección de estas anormalidades en el mismo procedimiento. Actualmente, se puede realizar como estudio inicial una angiografía por tomografía, con sensibilidades y especificidades similares al estándar de oro, que permite evidenciar la alteración vascular con una planeación terapéutica posterior (14).

El tratamiento se basa en el manejo por radiología intervencionista mediante angiografía y embolización, con sangre autóloga, coils, balón o cianoacrilato, que en casos seleccionados requiere el uso de stents vasculares. Este procedimiento tiene una baja morbimortalidad; es bien tolerado y exitoso en la mayoría de los pacientes. En muy pocos casos y como último recurso, la cirugía es el tratamiento para la hemobilia en la que se realiza la exploración directa del hígado, ligadura de vasos arteriales, resección del pseudoaneurisma, colecistectomía y resolución de la obstrucción de las vías biliares (15).

\section{CONCLUSIÓN}

La hemobilia debe sospecharse en los pacientes con evidencia de sangrado digestivo en el posoperatorio de cualquier procedimiento endoscópico, percutáneo o quirúrgico de hígado, vías biliares y páncreas. El abordaje inicial de estos pacientes es la angiografía por tomografía, que confirma el diagnóstico para planear un tratamiento angiográfico posterior, mientras que se reserva el manejo quirúrgico como última alternativa terapéutica. 


\section{REFERENCIAS}

1. Trombatore C, Scilletta R, Bellavia N, Trombatore P, Magnano S Lio V, Petrillo G, et al. Acute hemobilia from a pseudoaneurysm of the cystic artery arising from the left hepatic artery: Case report and literature review. Int J Surg Case Rep. 2017;37:60-64. https://doi.org/10.1016/j.ijscr.2017.06.014

2. Yopp A. Hemobilia and bilhemia. En: Blumgart LH (editor). Surgery of the liver, biliary tract and pancreas. 5. edición. Filadelfia: Elsevier; 2012. p. 1067-81. https://doi.org/10.1016/B978-1-4377-1454-8.00105-3

3. Baillie J. Hemobilia. Gastroenterol Hepatol (N Y). 2012;8(4):270-2.

4. Hidalgo F, Narváez JA, Reñé M, Domínguez J, Sancho C, Montanyà X. Treatment of hemobilia with selective hepatic artery embolization. J Vasc Interv Radiol. 1995;6(5):793-8. https://doi.org/10.1016/s1051-0443(95)71187-3

5. Chin MW, Enns R. Hemobilia. Curr Gastroenterol Rep. 2010;12(2):121-9. https://doi.org/10.1007/s11894-010-0092-5

6. Green MH, Duell RM, Johnson CD, Jamieson NV. Haemobilia. Br J Surg. 2001;88(6):773-86. https://doi.org/10.1046/j.1365-2168.2001.01756.x

7. Merrell SW, Schneider PD. Hemobilia--evolution of current diagnosis and treatment. West J Med. $1991 ; 155(6): 621-5$.

8. Sandblom P. Hemorrhage into the biliary tract following trauma; traumatic hemobilia. Surgery. 1948;24(3):571-86.
9. Luu MB, Deziel DJ. Unusual complications of gallstones. Surg Clin North Am. 2014;94(2):377-94. https://doi.org/10.1016/j.suc.2014.01.002

10. Yoshida J, Donahue PE, Nyhus LM. Hemobilia: review of recent experience with a worldwide problem. Am J Gastroenterol. 1987;82(5):448-53.

11. Gandhi V, Doctor N, Marar S, Nagral A, Nagral S. Major hemobilia--experience from a specialist unit in a developing country. Trop Gastroenterol. 2011;32(3):214-8.

12. Murugesan SD, Sathyanesan J, Lakshmanan A, Ramaswami S, Perumal S, Perumal SU, et al. Massive hemobilia: a diagnostic and therapeutic challenge. World J Surg. 2014;38(7):1755-62. https://doi.org/10.1007/s00268-013-2435-5

13. Casazza I, Guglietta MA, Argento G. Magnetic resonance cholangiopancreatography in the diagnosis of haemobilia. Case Rep Radiol. 2013;2013:792109. https://doi.org/10.1155/2013/792109

14. Prasad TV, Gupta AK, Garg P, Pal S, Gamanagatti S. Minimally invasive image-guided interventional management of Haemobilia. Trop Gastroenterol. 2015;36(3):179-84. https://doi.org/10.7869/tg.280

15. Cao H, Liu J, Li T, Cao G, Xu G, Zhai S, Xue J, Wang Z, Shi $S$, Bai W. Interventional therapy for the treatment of severe hemobilia after percutaneous transhepatic cholangial drainage: a case series. Int Surg. 2013;98(3):223-8. https://doi.org/10.9738/INTSURG-D-13-CC194 